\title{
Revisiting the Perceived Motion Model that Predicted 360 FPS Displays
}

\section{Scott Daly*}

Dolby Laboratories, Incorporated.

sdaly@dolby.com

A previous model of motion perception used in engineering display design predicted the need for a 360 FPS (frames per second) and 1080-line display and image signal format system, in order to match the viewing of reality. Now that 360 FPS gaming monitors are on the market, the model is revisited with twenty years of new studies, display technology advancements such as HDR (high dynamic range), and inclusion of new viewing tasks such as performance gaming. This revised model is then used to make new predictions for display and signal format requirements to achieve distortion-free viewing of reality and synthetic signals that do not impose limits to human performance.

CCS CONCEPTS • latency • gaming effects on visual psychophysics

Additional Keywords and Phrases: motion perception - spatiotemporal CSF • spatiovelocity CSF - eye movements • smooth pursuit • light adaptation • field of view (FOV) • latency • frame rate • frameless rendering

\section{Introduction}

The concept of the window of visibility [1] is a useful summary of the capability of the human visual system (HVS) capability to see spatiotemporal frequencies. The concept is commonly used in engineering for display design and video signal analysis and can be used to calculate the resolution and frame rate needed to match viewing of the real world. It is based on a model of the spatiotemporal contrast sensitivity function (ST-CSF) of the visual system, which is analogous to an MTF. The model has recently been expanded to include the effects of light adaptation, with the new version being called the pyramid of visibility [2]. However, the spatiotemporal CSF only characterizes one quadrant in the spatiotemporal Fourier plane, and thus cannot distinguish between counterphase flicker and motion.

Consequently, neither of these approaches include direct ability to describe motion, nor the capability of the human eye to track objects in motion. Object motion is often tracked by viewers using smooth pursuit eye movements, which has the mathematical consequences of reducing the object's retinal velocity (in $\mathrm{deg} / \mathrm{sec}$ ). This reduction in angular speed acts to reduce temporal frequencies on the retina, via the simple equation $\omega=\mathrm{v} \rho$ (temporal frequency = velocity $\times$ spatial frequency). Another model of the ST-CSF was developed more specifically toward motion, so that object motion and the eye tracking capabilities could be analyzed in a CSF framework [3,4]. It begins with a spatio-velocity model based on psychophysics [5] and modified for light adaptation to higher average luminance levels $\left(100 \mathrm{~cd} / \mathrm{m}^{\wedge} 2\right)$. It was later tested in a psychophysical experiment for four different conditions of motion tracking [6] and incorporated into a color

* contact info: sdaly@dolby.com 
video quality metric [7]. The key concept was to measure and model moving Gabor patterns ${ }^{1}$ of differing frequencies and characterize them in terms of velocity (temporal frequency could easily be calculated from the spatial frequency and velocity). A plot of the spatio-velocity CSF model fitting the data is shown in Fig 1. Importantly this data set was for a stabilized eye, so that means the effects of eye movements are removed from the model, so it can be thought of as a model of behavior from the retina and inward to further visual processing in the cortex.

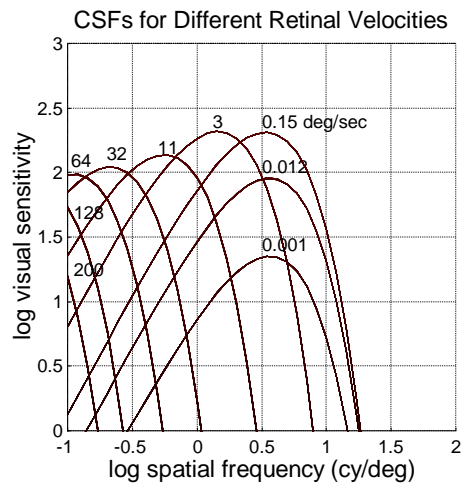

Figure 1. Key models: Spatial CSFs as a function of retinal velocity

While the eye movements are removed in the spatiovelocity CSF model, they are an important element for any practical display application. But there is an advantage in having the spatiovelocity model exclude the eye movements, because a separate model of eye movements gives more flexibility to model different applications. Of the various eye movements, including saccades, micro saccades, drift, and smooth pursuit, the smooth pursuit are the most relevant to frame rate. The smooth pursuit movements act to reduce the object velocities on the retina in a calculable manner by knowing the pursuit maximum velocity capability and gain (retinal slip). Figure 2 shows an eye tracking trace and dataset of smooth tracking [8].
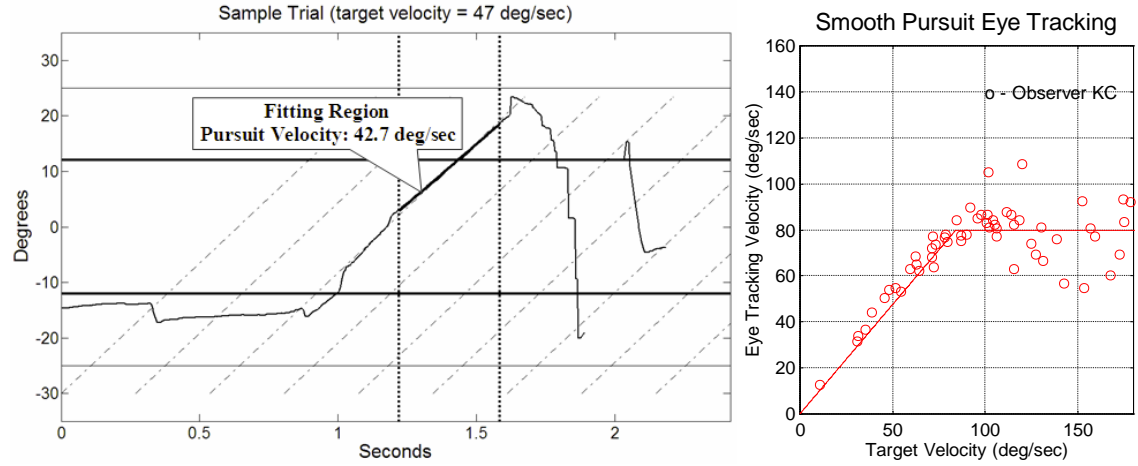

Figure 2: An eye tracking sample showing automatic determination of the smooth pursuit region and calculation of the pursuit velocity $(A)$ and the smooth pursuit capability including max speed before saccades intervene, data and model (B)

${ }^{1}$ initial work in [5] started with sinusoidal patterns 
The spatiovelocities of Fig. 1 have been replotted in a 2D contour plot and the max smooth pursuit capability $\&$ gain is used to reduce the velocities on the retina, and thus calculate the visible spatiovelocities in the scene space, which is also plotted in the figure. While inclusion of smooth pursuit capability has little effect on the low spatial frequencies in the plot, it can significantly extend the visibility of midrange spatial frequencies to higher velocities (e.g., at $1.0 \mathrm{log} c p d$ from $0.5 \mathrm{log}$ velocity to $1.2 \mathrm{log}$ velocity, i.e., at $10 \mathrm{cpd}$ from 3.1 to $16 \mathrm{deg} \mathrm{sec}$ in linear units). This represents the best capability of the visual system. Thus, this usage is consistent with worst-case engineering design principles.
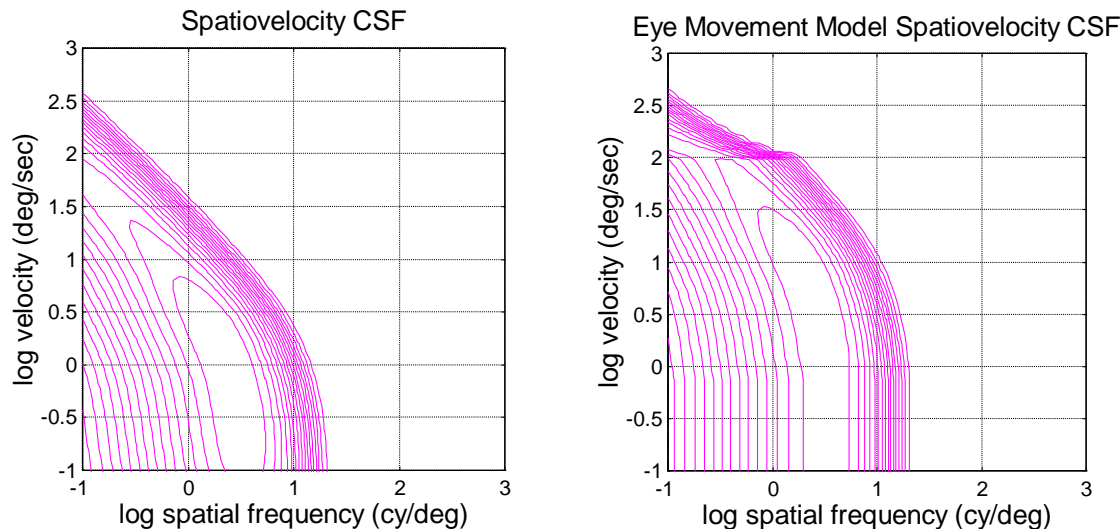

Fig 3 Spatiovelocity CSF model of Fig 1 replotted into spatiovelocity plane (L) Effect of smooth pursuit eye movement model on the spatiovelocity CSF (R).

For engineering design of either displays and or signal formats, a spatiovelocity CSF is not very helpful. Rather, what is needed is a spatiotemporal representation. Fortunately, the spatio-velocity CSF can be rotated into a spatiotemporal CSF, for either the case of excluding or including the effects of the pursuit eye movements. The outer contours are the highest frequency that can be seen (with spatiotemporal coordinates), and is analogous to the window of visibility mentioned in the intro. The consequence of adding the eye movement model is to increase the temporal frequency capability of the visual system, and thus increase the demands on display or signal format design. It is this extension of the visibility to higher temporal frequencies that cannot be done with a spatiotemporal CSF model, such as the window of visibility model derived directly from spatial and temporal frequencies. 

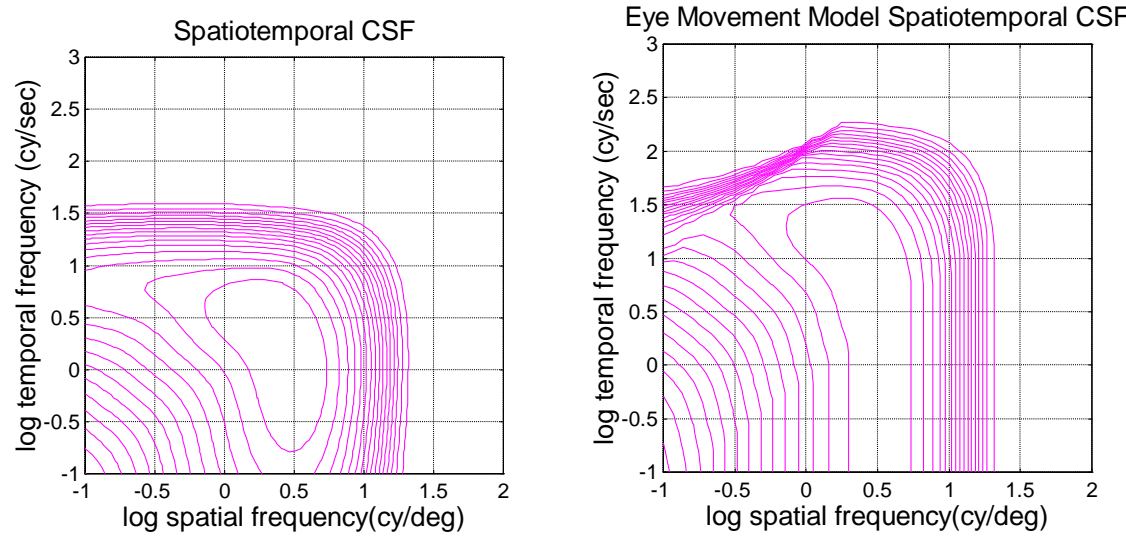

Fig 4 Effect of eye movement model on spatiovelocity CSF

For example, in the original Window of Visibility model, the max visible spatial frequency is $30 \mathrm{cpd}$ (cy/deg), which requires $60 \mathrm{pix} / \mathrm{deg}$ sampling, which corresponds to 1080p for 3 picture heights viewing (3H). The maximum visible temporal frequency is $30 \mathrm{cy} / \mathrm{sec}$, which requires $60 \mathrm{~Hz}$ sampling. Thus, a 1080p display at 60 FPS when viewed at $3 \mathrm{H}$ would capture all that the HVS could perceive. However, the model based on the spatiovelocity CSF combined with eye movements predicted that 360 FPS and 1080p would be needed, a much costlier system. This result is shown below in Fig. 5 [4] and both axes are log values. The arrow points to the spatial frequency having the highest temporal frequency, which essentially sets the necessary temporal sampling rate, and can be thought of as a critical spatial frequency.

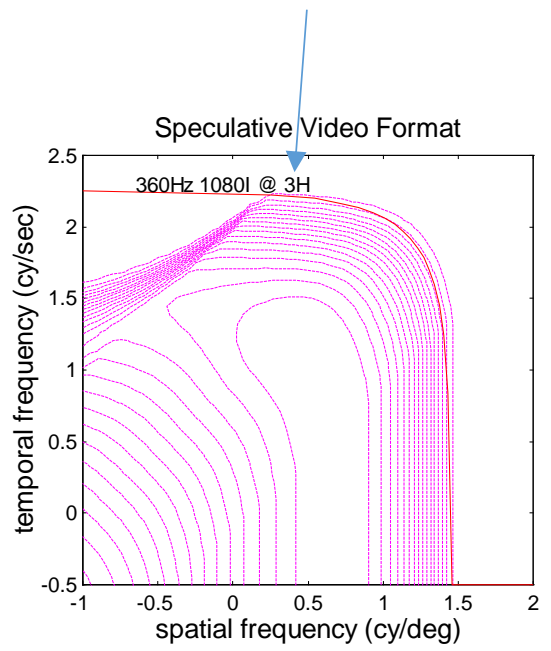

Figure 5. Speculated video/display format that closely matches visual limits circa $2000\left(\mathrm{LA}=100 \mathrm{~cd} / \mathrm{m}^{\wedge} 2\right)$

At the time of that prediction (turn of the century), there were only a few research displays on the planet with frame rates exceeding that prediction, and none with that high of a frame rate and that predicted resolution. Now there are several gaming monitors on the market with that exact frame rate and spatial resolution capability. 


\section{REVISTING THE MOTION MODEL AND NEW DESIGN PREDICTIONS FOR GAMING}

This work will revisit that model, and update it for current display and signal capability, as well as considering the impact of latency issues, relevant to gaming. The new model can be used to predict a new design goal. In the twenty years since that work, there have been several key papers and technological advancements that can be incorporated into that spatiovelocity model, and that would result in an update of the specs. There are three main effects considered that would be likely to affect the key display parameters of frame rate, resolution, and bit-depth. These are light adaptation, improved eye tracking models, and effect of gaming experience.

\section{Light adaptation}

One key change in the capabilities of displays is due to HDR, operating at much higher max luminance levels with possible higher average luminance levels. Much of early HDR video content (particularly broadcast) tends to keep the diffuse reflective regions about the same, using the extra dynamic range to render highlights (specular reflections and self-luminous regions) and extending dark detail. Due to the generally smaller regions of highlights, the average luminance increases only slightly, and the model described in the intro should be applicable as it was tuned for a $100 \mathrm{~cd} / \mathrm{m}^{2}$ light adaptation level. However, another approach to HDR is to use the extra range for scene-to-scene dynamic range changes [9], so that a sense of realism in going from night to indoor to daylit scenes occurs. The different types of dynamic range are referred to an intrascene dynamic range and interscene dynamic range. The interscene DR is particularly relevant to gaming as the player goes from environment to environment and their duration within any environment is unpredictable (thus they can have the ability to fully adapt to that light level). In addition, once the field of view (FOV) of the scene is large enough, local adaptation must be considered [10,11]. This consideration of variable light adaptation, also called 'floating adaptation' both across time and spatial location has been a key feature of both a current HDR image format [12] and the HDR image quality model, known as HDRVDP\# [13].

When they occur, these higher average luminance levels can cause higher light adaptation levels in the viewer, which is known to result in higher spatial and temporal frequency visibilities, as can be seen in Fig.6. Since the previous model was designed for $100 \mathrm{~cd} / \mathrm{m}^{2}$ it is very close to the best spatial CSF behavior which asymptotes near $1000 \mathrm{~cd} / \mathrm{m}^{2}$. By today's standards, it seems unlikely that average luminance levels of $1000 \mathrm{~cd} / \mathrm{m}^{2}$ would be likely to occur, but this is mainly an issue of luminance inefficiency of display systems and viewing conditions. In consideration of sustained local regions of high luminance (such as a sky) or effective use of interscene dynamic range, it is possible that adaptation levels of $1000 \mathrm{~cd} / \mathrm{m}^{2}$ are possible, and thus may affect calculations of upper bounds. Regarding temporal frequency, the plot in Fig 6 is the maximum visible temporal frequency (CFF= critical fusion frequency) as a function of light adaptation level, known as the Ferry-Porter law for its linear dependence on the log of light adpattaion level. Five different eccentricities are shown, and the data shows no indication of an asymptote, but the plot is only measured to $500 \mathrm{~cd} / \mathrm{m}^{2}$ (3000 Troland, $\left.\mathrm{Td} \sim 500 \mathrm{~cd} / \mathrm{m}^{2}\right)$. Regardless, there is substantial increase from $100 \mathrm{~cd} / \mathrm{m}^{2}$ to 500 $\mathrm{cd} / \mathrm{m}^{2}$ (1000 to $\left.3000 \mathrm{Td}\right)$. 

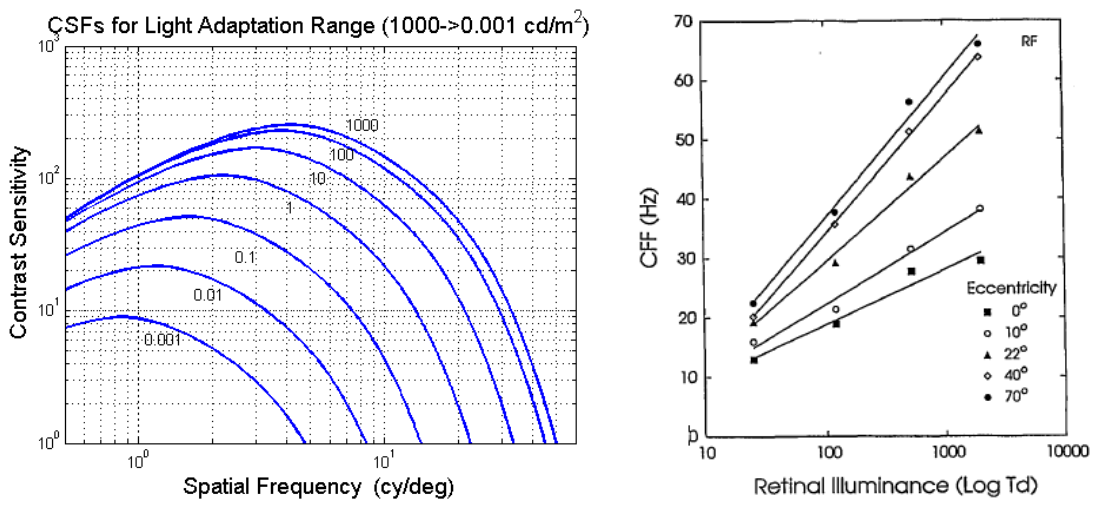

Fig. 6. Two key light adaptation effects: spatial Contrast Sensitivity Function as a function of light adpattaion levels (L) and maximum visible temporal frequency as a function of luminance adaptation level $(R)$.

We developed a new spatiovelocity model that enables these light adaptation effects to be specifically modelled. While there exists no full spatiovelocity data set as a function of light adaptation level, we can design a model by constraining the spatiovelocity CSF at 100 nits to match that of the previous model, constrain the results spatial CSF to match the model shown in Figure 6A, as well as constraining it to have the cut-off temporal frequency follow the Ferry-Porter law of figure 6B. A Lagrangian cost function was used with the downhill simplex method [13] to make this optimization. The new model's fit to the Ferry-Porter law as well as the original spatiovelocity CSFs at light adaptation level of $34 \mathrm{~cd} / \mathrm{m}^{\wedge} 2$ are shown in Fig 7 . A spatiovelocity contour plot for light level going from 1.0 to $1000 \mathrm{~cd} /{ }^{\wedge} 2$ is shown in Fig. 8. Both the increase in maximum visible spatial frequency as evidenced in the light adaptive CSF (Fig 6) and the increase in visible velocities in correspondence with the increased in temporal frequency visibility according to the Ferry-Porter law can be seen in these plots as the light adpattaion level increases. Details of the model and more results are in [15].
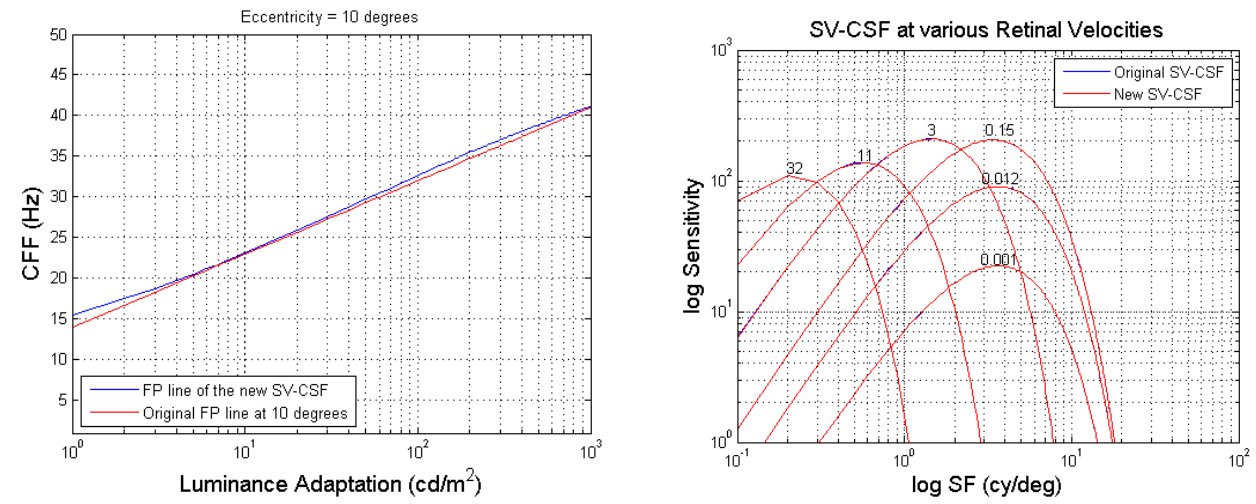

Fig. 7. New model as constrained by the Ferry Porter law showing the relation between the cutoff flicker frequency (CFF) and log of luminance adaptation level of the new SV-CSF (L). Spatiovelocity CSF as a function of retinal velocity for both the original SV-CSF and the new SV-CSF at $L A=34 \mathrm{~cd} / \mathrm{m}^{2}$. Note the near perfect match between the original SV-CSF and the new SV-CSF at this luminance adaptation level and at various retinal velocities. The numbers on top of each curve show the relevant retinal velocity $(\mathrm{deg} / \mathrm{sec})(\mathrm{R})$. 

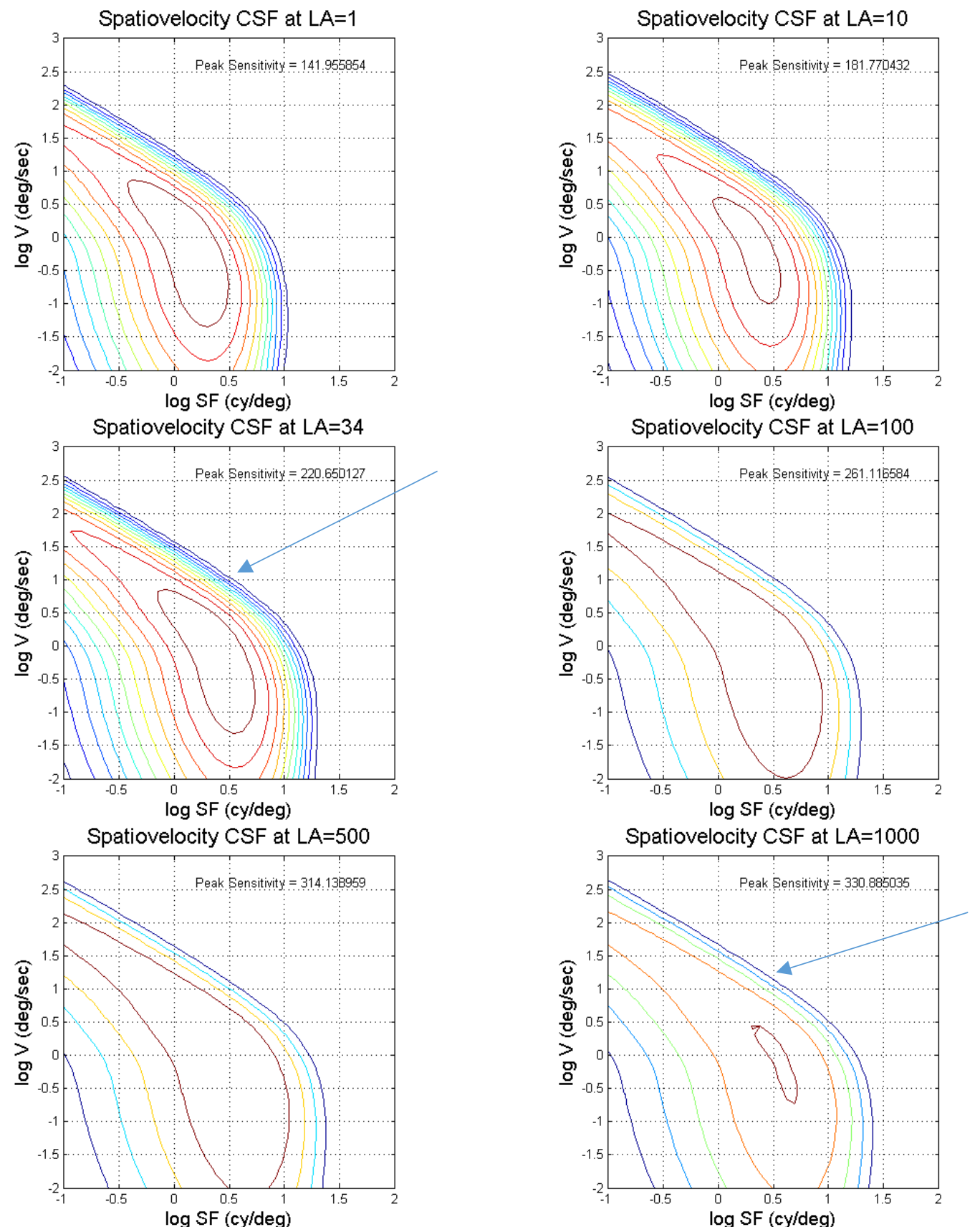

Fig. 8. Contour plots of the new spatiovelocity CSF at six different luminance adaptation (LA) levels 1,10, 34, 100, 500, and $1000 \mathrm{~cd} / \mathrm{m}^{2}$. Blue arrows correspond to the CSF's peak spatila frequency at most light levels (3cpd)

As mentioned in the introduction, it is necessary to rotate the spatiovelocity CSF into the spatiotemporal domain for the purposed of making engineering design calculations, such as resolution and 
frame rate. Cross sectional plots of the resulting spatiotemporal CSF are shown in Figure 9, plotted for each of the frequencies having maximum overall sensitivity in the orthogonal domain. From these plots we can make simple estimates of new resolution and frame rate increases due to the increased light adaptation capabilities with HDR systems. We will focus these calculations on the differences between $100 \mathrm{~cd} / \mathrm{m}^{2}$ and $1000 \mathrm{~cd} / \mathrm{m}^{2}$. Regarding the spatial resolution criteria, we have the max visible spatial frequency (cut-off frequency) at $100 \mathrm{~cd} / \mathrm{m}^{2}$ being approximately $20 \mathrm{cpd}$ in the spatial CSF plot (L), while the cutoff for 1000 $\mathrm{cd} / \mathrm{m}^{2}$ is approx. $25 \mathrm{cpd}$. So, the gain in resolution as needed to go from 100 to $1000 \mathrm{~cd} / \mathrm{m}^{2}$ is 1.25 . Similarly, for the frame rate criteria, we see from the temporal CSF (R) that the max temporal frequency increase from $35 \mathrm{~Hz}$ to $50 \mathrm{~Hz}$, as the light adaptation level increase from 100 to 1000, for a gain of 1.43 . This is for $3 \mathrm{cpd}$, which is the spatial frequency corresponding to the blue arrow in Fig. 5. These gains for spatial frequency and temporal frequency will be used in the table in the section on Combined Results.
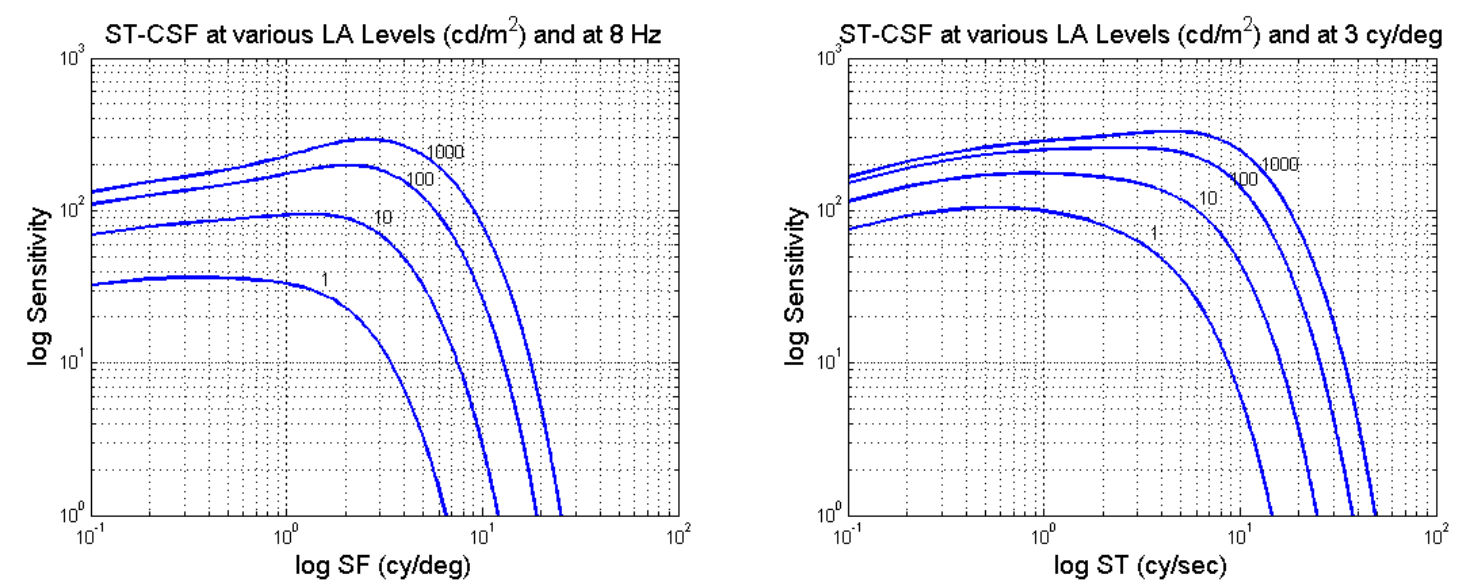

Figure 9: cross sectional slices of the resulting spatiotemporal CSF from the new light adaptative spatio velocity CSF. The series of blue curves are for different light adpattaion levels. The spatil CSF is plotted for the temporal frequency having the maximum sensitivity, which ais $8 \mathrm{~Hz}$. (L). Similarly, the temporal CSF is plotted for the peak spatial frequency, which is $3 \mathrm{cpd}$.

\section{Improved Eye Tracking}

Another candidate factor that may increase the demands from the 2000 era prediction is improved eye tracking capability. This can show up in terms of FOV and stimulus properties. As previously mentioned, a key factor is the maximum smooth pursuit velocity and tracking gain. The values used in the previous model were from the experiment at that time that gave the best performance ( $80 \mathrm{deg} / \mathrm{sec}$ max tracking, gain $=0.9$ ). It is already known that the FOV can have an effect on this value. For example, earlier experiments with more limited FOV showed max values of 30-40 cy/deg [16]. In addition, there might be an effect of the type of stimulus that is being tracked, such as its angular size and content. For example, both eye tracking studies mentioned used a small spot of light as the tracking stimulus. 


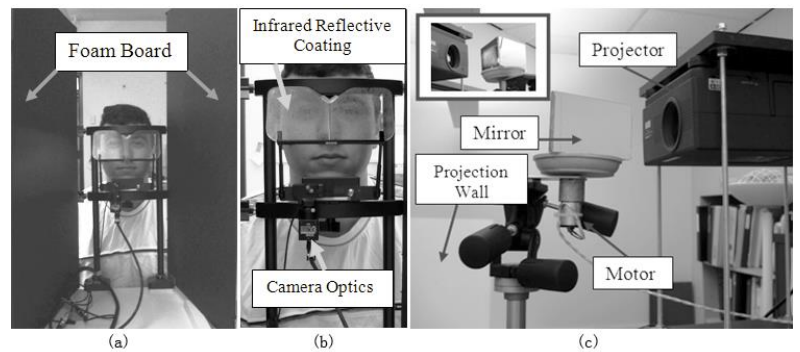

Figure 10: Experimental apparatus for updated eye tracking experiment. FOV was $120 \mathrm{deg}(+/-60 \mathrm{deg})$ and the stimulus was driven by a motorized mirror to avoid digital sampling effects.

A post-2000 experiment that studied smooth pursuit training for both a wide FOV and different types of stimulus [17] was performed, and in this section, we consider how those results may impact the display design specs of frame rate. In addition, that experiment used more advanced equipment, as well as automated techniques for extracting smooth pursuit behavior from the eye tracking data traces, which were lacking in the earlier data. The set-up is shown in Fig 10, and the stimuli included a dot ( $0.1 \mathrm{deg})$ to compare to previous studies, an apple for having a more realistic object of interest and wider range frequencies being present having size ( $2 \mathrm{deg}$ ), and flower scene of size $17 \mathrm{deg}$. A hypothesis is that wider stimulus size may recruit motion or spatiotemporal signals from the parafovea and periphery to enable better performance, as well as multiple frequencies, texture or recognizability. A chin rest was used to prevent OKR (opto-kinetic reflex) head movements, and to enable engineering design for applications where the aspects of head movements and eye movements can either both be measured or designed with specific condition.
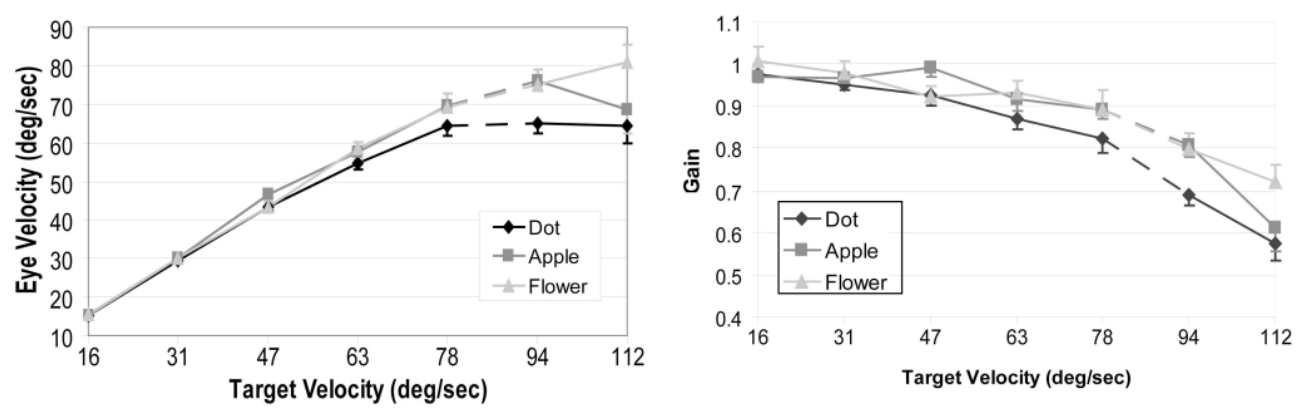

Figure 11: Data from updated smooth pursuit eye tracking experiment. Plots are compiled over two different experiments, with the speeds greater than 78 using updated equipment.

The results show improvement performance for larger stimuli (Flower $17 \mathrm{deg}>$ Apple $2 \mathrm{deg}>$ Dot $0.1 \mathrm{deg}$ ) for both maximum velocities and gain, but there is no significant improvement due to a wider FOV (e.g., compare the dot results to previous studies. ) For updating the display design predictions we can compare the previous eye tacking model based on a small spot stimulus ( $80 \mathrm{deg} / \mathrm{sec}$ max with a gain of 0.9 giving a retinal tracking of $72 \mathrm{deg} / \mathrm{sec}$ ) with data obtained from the plots of Fig 11 for the large object 
stimulus of $17 \mathrm{deg}$ ( $112 \mathrm{deg} / \mathrm{sec}$ with a gain of 0.7 giving a retinal tracking of $80 \mathrm{deg} / \mathrm{sec}$ ). Comparing these to numbers, we calculate an increase in eye tracking capability of $80 / 72=1.11 x$. This value will be used in the table below for consideration of the combined effects. Another consideration on frame rate concerns saccades, as it has been found that vision is not 'blanked' during saccades as was the common understanding, but merely reduced in sensitivity. Demonstrations of full color visibility during saccades have been presented at SIGGRAPH [18], for example. Extreme high temporal frequency designs, referred to as frameless rendering, have been motivated by saccadic eye movements, as well as latency issues [19]. While it has been demonstrated that image signals can be perceived during a saccade, these were under very special considerations that generally don't occur with hold-type displays. However, they may be relevant for bright and sparse duty cycle PWM display systems. Such considerations are beyond the scope of this paper, but worth keeping in mind for display systems that are more advanced than hold-type.

\section{Gaming aspects}

Much of the applied vision science work has been motivated by still images and video (print and TV), and certain assumptions have followed along with those applications. Computer game users engage with the display in a much more active manner than the more passive interactions common to video and TV watching. In addition, there has been a higher level of enthusiasm in customizing equipment for either performance or immersion. These two factors may affect the display in terms of immersion and neurophysiological changes resulting from accumulation of gameplay experience.

For the immersion aspect, as an example it is quite common that PC gamers use more than two screens to enable a wider FOV. In addition, gaming is one of the larger drivers of VR, which has increased FOV as one its prime differentiators from traditional displays. For example, while digital TV has evolved from a viewing distance of 3 pictures height $(3 \mathrm{H}$ ) having a 15 deg FOV to $1.6 \mathrm{H}$ and $\sim 63$ deg FOV for UHDTV (and 0.8 and $\sim 90$ deg FOV for upcoming 8k displays ), VR gaming is already in the process of evolving from 110 deg FOV to a goal of complete visual immersion ( $200 \mathrm{deg}$ ). The expanded FOV requires higher resolution when expressed in total pixels, and this will be included in the table in a straightforward manner assuming the max frequency in cpd doesn't change with FOV, and that eye movements are capable everywhere within the displayed FOV, and lastly that no foveated rendering is used. This last point is currently true for many current VR/AR display systems but is unlikely with future systems.

Less well-known are the effects of gameplay experience on the essentials of neurophysiological processing. It is expected that reflex times may improve, as with all sports involving rapid decisions with moving (visual) objects. Less expected are the findings that essential detection changes can occur, as found in careful studies involving both experienced gamers, non-gamers, and trained non-gamers. One study looking at the durations of the effects showed they can last over 5 months [20]. One of two key changes relating to display design involve overall sensitivity effects. In one paper [21], the experienced gamers showed an increase in sensitivity of $1.78 \mathrm{X}$, and a critical duration improvement of $1.38 \mathrm{X}$ (a reduction in time by $1 / 1.38 \mathrm{X}$ ). In another study focused on training paradigms as opposed to experienced gamers, the sensitivity boost was slightly smaller, at 1.3X [22]. In both studies, the boost was strongest at the frequencies near the peak of the CSF, which is consistent with reductions in internal noise [23], a low-level neurophysiological effect, such as might occur in layer V1 of the primary visual cortex. It has also been modeled as an enhancement in the connection strength between the layer providing the sensory experience, 
and the layer integrating the evidence [24], which would be a much higher-level effect. Locations for such would be in the posterior parietal cortex where stimuli integration and consolidation occur or the prefrontal association cortex where higher level decisions that inform the premotor cortical regions. This model has an advantage in predicting decreased reaction times. Both explanations are based on concepts of neural plasticity.

Additionally, some studies have found increases in acuity and peripheral processing. Acuity is related to the spatial frequency cut-off and hence affecting required display resolution. Gamer effects on acuity are reported in two studies $[20,22]$. But acuity increases are also a consequence of simply increasing the overall sensitivity as found in these and other studies. This is a consequence because the CSF decays with frequency at a certain slope, so elevating sensitivity has a side effect of increasing the cut-off frequency. In addition, the acuity increases are strongest for subjects whose acuity is worse than from 20/20 vision, and the 'gamer acuity' effect diminishes for subjects with 20/20 vision. For the purposes of this engineering design model, we will assume there are no separate acuity effects that are not the side effect of a sensitivity increase, which will be modelled. There is also improved peripheral processing for those with significant gaming experience, in terms of crowding thresholds [20], which relates to attention control. This factor is hard to map to specific display applications, unless foveated rendering is used, which is out of scope. So, peripheral effects will not be used here in updating display and signal format estimates. Lastly, as latency is a well-known factor for both active gaming and passive VR experiences, there has been found a gamer effect [24]. The work found a dependency on the effect, which was stronger for uncertain stimuli than more certain stimuli. For a motion discrimination study, they found a reduction of latency from $900 \mathrm{~ms}$ to $600 \mathrm{~ms}$ for highly uncertain stimuli, giving a reduction ratio $600 / 900=0.67$, requiring a $1 / 0.67$ or $1.5 x$ improvement in latency specs. For stimuli that were more certain (less stimuli noise), the reduction went from $570 \mathrm{~ms}$ to $450 \mathrm{~ms}$, giving a ratio of $450 / 570=.789$. Keep in mind the latencies with a 60 FPS display are about $17 \mathrm{~ms}$, while a 360 FPS display would have a minimum latency of $2.8 \mathrm{~ms}$. Since both of these gamer latencies for this particular psychophysical task are well above the latencies caused by frame rates, and well above what is known for head \& eye movement latencies in VR/AR ( 15 ms), we will ignore the gamer effects on latency in the design specifications.

\section{New Speculative signal/display format}

We will combine these key effects to update the display and signal format predictions using the newer model. There are no experiments testing interaction of light adaptation, eye movements, and the neural plasticity found in the gamer effects, but there is no direct evidence that such interactions would occur. This is because light adaptation is primarily a retinal effect, eye movements capability is not expected to be affected by light adaptation nor pupil size, except at the dark end where the signal detection is reduced by photon noise. It is the bright end of light adaptation that pushes the system requirements. So, the new specifications/predictions assume simple multiplicative effects, no suboptimal combinations, no masking type effects, and no facilitation across the effects.

Not discussed in detail, but there has been discussion in the display community that the $30 \mathrm{cy} / \mathrm{deg}$ cutoff frequency assumption used for decades in digital TV is too low, and the most cited reasons are that viewers can be better than 20/20, the spatial cut-off frequency increases with light adaptation, and hyperacuity. The relevance of hyperacuity for different display applications has recently been discussed [25], 
with key summary that a $30 \mathrm{cy} / \mathrm{deg}$ cutoff is appropriate for optically captured and natural real-world signals, while the $60 \mathrm{cy} / \mathrm{deg}$ cutoff from hyperacuity is limited to optotype (a signal classification that includes text and graphical line art) and other signals that approach test targets. Of these, we will only consider the effect of light adaptation on the resolution criterium. Since none of these newly considered affects are larger than $2 X$, they may be considered subtle. However, their combination leads to specs that are indeed very daunting or drooling, depending on whether the reader is an engineer or a gamer.

Also, listed in the table but not discussed is the bit-depth increase needed for HDR. Most of this effect is due to the range increase, but there is a factor caused by better SNR capture with high-end digital cinema cameras. In one of the studies leading to the SMPTE $2048 \mathrm{spec}$ for a range of $0-10,000 \mathrm{~cd} / \mathrm{m}^{2}$, it was found that 10 bits was needed for optically captured content and natural imagery, while 11 bits was needed for synthetic test targets [26]. For the table, we only list the case for the 10 bits requirement.

Table 1: Updated Speculations on Display Format to match perception of reality

\begin{tabular}{|c|c|c|c|c|c|}
\hline & $\begin{array}{c}\text { Speculations } \\
\text { Circa } 2000\end{array}$ & $\begin{array}{l}\text { HDR Effects } \\
\text { (light adaptation) }\end{array}$ & $\begin{array}{c}\text { FOV } \\
\text { (\& stimuli size effects) }\end{array}$ & $\begin{array}{c}\text { Gamer } \\
\text { Experience } \\
\text { (plasticity } \\
\text { effects) } \\
\end{array}$ & $\begin{array}{l}\text { Combined } \\
\text { New } \\
\text { Speculation }\end{array}$ \\
\hline $\begin{array}{l}\text { Frame } \\
\text { rate }\end{array}$ & 360 FPS & $\begin{array}{l}515 \text { FPS } \\
1.43 \mathrm{X}\end{array}$ & $\begin{array}{l}400 \mathrm{FPS} \\
1.11 \mathrm{X}\end{array}$ & $\begin{array}{l}495 \text { FPS } \\
1.38 \mathrm{X}\end{array}$ & $\begin{array}{l}790 \text { FPS } \\
2.2 \mathrm{X}\end{array}$ \\
\hline $\begin{array}{l}\text { Bit - } \\
\text { depth }\end{array}$ & $8 \mathrm{RGB}$ & $\begin{array}{l}10 \text { RGB } \\
4 \mathrm{X}\end{array}$ & 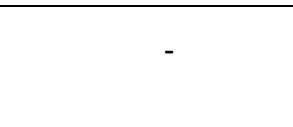 & $\begin{array}{l}9 \text { RGB } \\
1.78 \mathrm{X}\end{array}$ & $\begin{array}{l}11 \text { RGB } \\
7 \mathrm{X}\end{array}$ \\
\hline Resolution & $\begin{array}{l}\text { 1920x1080i } \\
@ 3 \mathrm{H} \\
30 \mathrm{cpd} \text { acuity } \\
@ 100 \mathrm{~cd} / \mathrm{m}^{\wedge} 2 \\
\\
60 \text { cpd for } \\
\text { hyperacuity }\end{array}$ & $\begin{array}{l}\text { 2400x1350@3H } \\
1.25 \mathrm{X}\end{array}$ & $\begin{array}{l}\text { 3840x2160@1.6H } \\
\text { 60deg FOV } \\
7680 x 4320 @ 0.8 \mathrm{H} \\
\text { \%0deg } \\
\text { 12,000x8000 VR } \\
200 \text { deg -non-foveated } \\
\\
2 \mathrm{X}->8 \mathrm{X} \\
\text { accordingly }\end{array}$ & 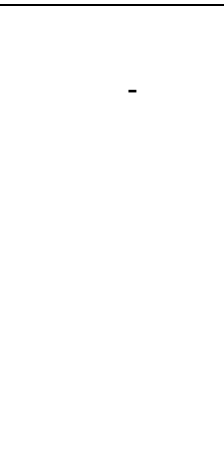 & $\begin{array}{l}9600 \times 5400 \\
@ 0.8 \mathrm{H}\end{array}$ \\
\hline Latency & none & $?$ & $?$ & $\begin{array}{c}1.5 \mathrm{X} \\
\text { (improvement) }\end{array}$ & \\
\hline
\end{tabular}

While latency is a key study area in gaming, we found that current latencies are due to bottlenecks other than the display frame rate and signal format. However, deriving latencies from frame rates neglects to consider the display materials temporal response, which varies from LCD type and OLED driving method, such details being out of scope for this paper. In general, the latency is not a consequence of display design, but rather more upstream processing (GPUs and streaming). So, we only list the gaming effect latency 
improvement specification in the table. Of course, the main point of this work is not to make a prediction, but rather to clarify the key effects and how they contribute to design goals.

\section{REFERENCES}

[1] Watson, Ahumada, Farrell (1986) window of visibility: a psychophysical theory of fidelity for time sampled visual motion displays. JOSA A V 3

[2] Watson and Ahumada (2016) The pyramid of visibility. JOV 16(12)

[3] Daly (1998) S. Daly (1998) "Engineering Observations from spatiovelocity and spatiotemporal visual models. SPIE Electronic Imaging. SPIE Proceedings V. 3299, pp 180-193.

[4] Daly (2001) "Engineering observations from spatiovelocity and spatiotemporal visual models", Chapter 9 in Vision Models and Applications to Image and Video Processing, ed. C. J. van den Branden Lambrecht, Kluwer Academic Publishers.

[5] Kelly (1979) "Motion and vision. III. Stabilized spatio-temporal threshold surface" JOSA 69, pp. 1340-1349.

[6] J. Laird, M. Rosen, J. Pelz, E. Montag, and S. Daly (2006) Spatio-velocity CSF as a function of retinal velocity using unstabilized stimuli SPIE-IS\&T V. 6057

[7] K. Hirai, J. Tumurtogoo, A. Kikuchi, N. Tsumura, T. Nakaguchi, and Y. Miyake (2010) IEICE Trans. Inf. \& Syst. V. E93-D \#5

[8] Meyer, A. G. Lasker, and D. A. Robinson (1985) “The upper limit of human smooth pursuit velocity” Vis. Res. 25, pp. 561-563.

[9] S. Ruggieri (2016) Breaking outside of the 100-nit box - a colorist' view to HDR Grading. SMPTE Annual technical conference DOI: $10.5594 / \mathrm{M} 001690$

[10] W. Makous, D. Macleod, and D. Williams (1985) Local adaptation ARVO.

[11] P. Van Gorp, K. Myszkowski, E. Graf, ad R. Mantiuk (2015) A model of local adaptation. ACM TOG 34(6)

[12] Timo Kunkel, Robert Wanat, Jaclyn Pytlarz, Robin Atkins, \& Scott Daly (2018) “Assessing Color Discernibility in HDR Imaging using Adaptation Hulls" CIC (Color Imaging Conference), Vancouver, Canada

[13] R. Mantiuk, S. Daly, K. Myszkowski, H. Seidel, (2005) Predicting Visible Differences in high-dynamic-range images: model and its calibration. SPIE proc 5666, Electronic Imaging Conference: Human Vision and Electronic Imaging, pp 204-214.

[14] J. A. Nelder and R. Mead, "A simplex method for function minimization," The Computer Journal, vol. 7, no.4, pp. 308-313, 1965.

[15] H. Hadizadeh and S. Daly (2011) Spatiovelocity Contrast Sensitivity Function as a function of luminance adaptation level. DolbyCanada Technical Report. In submission stages for external publication

[16] G. Westheimer (1954) "Eye movement responses to a horizontally moving visual stimulus", AMA Archives of Ophthalmology, pp932-941

[17] F. Li, Pelz, Daly (2010) effects of stimulus size and velocity $n$ steady-state smooth pursuit induced by realistic images. Proc. SPIE V. 7527

[18] H. Ando and J. Watanabe (2007) Full-scale saccade-based display : public/private image presentation based on gaze-contingent visual illusion SIGGRAPH Aug. https://doi.org/10.1145/1278280.1278286

[19] Greer, Spjut, Luebke, and Whitted (2016) Hybrid modulation for near zero latency. SID Display week 8-3.

[20] C. Green, R. Li, D. Bavelier (2010) perceptual learning during action video game playing. Topics in cognitive neuroscience V2.

[21] R. Li, U. Polat, W. Makous, and D. Bavelier (2009) enhancing the contrast sensitivity function through action video game training. Nat. Neurosci. May 12(5)

[22] J. Deveau, G. Lovick, and A. Seitz (2014) broad-based visual benefits from training with an integrated perceptual-learning video game. Vis Res. 99, 134-140 (training effects with clinical 'game')

[23] S. Daly (1990) “Application of a noise-adaptive contrast sensitivity function to image data compression” Optical Engineering V. 29 (8) 977-987.

[24] C. Green, A. Pouget and D. Bavelier (2010) Improved probabilistic inference as a general learning mechanism with action video games Current Biology 20, 1573-1579

[25] S. McCarthy, S. Daly, and T. Kunkel (2019) "Framework for evaluating display resolution and size in the context of video compression and visual acuity". SID Display Week. Paper 79-1.

[26] S. Miller, S. Daly, and M. Nezamabadi (2013) Perceptual signal coding for more efficient usage of bit codes, SMPTE MIJ. 